\title{
AN ANALOGUE OF A RESULT OF JACOBSTHAL
}

\author{
by ECKFORD COHEN \\ (Received 14th February 1962)
}

Dedicated to Professor Jacobsthal on his eightieth birthday

\section{Introduction}

Jacobsthal (4) has proved that the $n \times n$ matrix

$$
A=([r / s]), r, s=1,2, \ldots, n,
$$

is invertible with the inverse,

$$
A^{-1}=(\mu(r / s)-\mu(r /(s+1))), r, s=1,2, \ldots, n .
$$

Here $\mu(x)$ denotes the Möbius function for positive integral $x$ and is assumed to be 0 for other values; $[x]$ has its usual meaning as the number of positive integers $\leqq x$.

Carlitz (1) has given a particularly simple proof of this result, starting from the identity,

$$
\sum_{d \backslash n} \mu(d)=\in(n) \stackrel{\text { def }}{=}\left\{\begin{array}{l}
1(n=1) \\
0(n>1) .
\end{array}\right.
$$

In this note we prove a " unitary " analogue of Jacobsthal's result, contained in (7) below. The proof is based upon the analogue (5) of (3) for unitary divisors. We indicate in $\S \mathbf{3}$ a generalisation from which several special results are then drawn $(\S 4)$.

\section{An Analogue of (2)}

We say that $d$ is a unitary divisor of $n$, written $d \| n$ or $d^{*} \delta=n$, provided $n=d \delta,(d, \delta)=1$; in such a case, we also call $n$ a unitary multiple of $d$. Let us place $\mu^{*}(n)=\mu(v(n))$ where $v(n)$ is the maximal square-free divisor of $n$ and define

$$
\mu^{*}(r ; s)= \begin{cases}\mu^{*}(\dot{r} / s) & \text { if } s \| r \\ 0 & \text { otherwise. }\end{cases}
$$

We recall from $(2,(2.5))$ that

$$
\sum_{d \| n} \mu^{*}(d)=\in(n) .
$$

In addition, let us define $[x, s]$ to be the number of unitary multiples of $s$ not exceeding $x$. 
We prove now that the matrix

has the inverse

$$
A^{*}=([r, s]), \quad r, s=1,2, \ldots, n,
$$

$$
\left(\delta_{r s}\right) / A^{*}=\left(\mu^{*}(r ; s)-\mu^{*}(r ; s+1)\right), \quad r, s=1, \ldots, n,
$$

$\delta_{r s}$ denoting of course the Kronecker delta function.

Letting $\rho(n)$ denote the function with the value 1 for all $n$, we place

$$
\rho^{*}(r ; s)= \begin{cases}\rho(r / s)=1 & \text { if } s \| r, \\ 0 & \text { otherwise. }\end{cases}
$$

One obtains from (4) and (8), if $s \| r$,

$$
\begin{aligned}
\sum & \equiv \sum_{k=1}^{n} \mu^{*}(r ; k) \rho^{*}(k ; s)=\sum_{\substack{k\|r \\
s\| k}} \mu^{*}(r / k) \quad \ldots \ldots \ldots \\
& =\sum_{\substack{s^{*} s^{s}=k \\
k^{*} d=r}} \mu^{*}(d)=\sum_{\substack{d \| r \\
d^{*} s^{\prime}=r / s}} \mu^{*}(d)=\sum_{d \| r / s} \mu^{*}(d) ;
\end{aligned}
$$

since $\Sigma$ is 0 in case $s \nVdash r$, it follows from (5) that

$$
\sum=\sum_{k=1}^{n} \mu^{*}(r ; k) \rho^{*}(k ; s)=\delta_{r, s}(r, s=1,2, \ldots, n) .
$$

Noting that

$$
\sum_{k=1}^{r} \rho(k ; s)=[r, s]
$$

one finds on applying partial summation that

$$
\sum=\sum_{k=1}^{n}\left(\mu^{*}(r ; k)-\mu^{*}(r ; k+1)\right)[k ; s]+[n, s] \mu^{*}(r ; n+1),
$$

so that (10) yields

$$
\sum_{k=1}^{n}\left(\mu^{*}(r ; k)-\mu^{*}(r ; k+1)\right)[k, s]=\delta_{r, s}(r, s=1,2, \ldots, n) .
$$

This is equivalent to the desired result.

From (11) we have

$$
\rho(r ; s)=[r, s]-[r-1, s] ;
$$

hence (10) may be put into the matric form,

$$
\left(\mu^{*}(r ; s)\right)([r, s]-[r-1, s])=\left(\delta_{r s}\right), r, s=1,2, \ldots, n .
$$

\section{A Generalisation}

For arbitrary arithmetical functions $f(n)$, we write

$$
f(r ; s)=\left\{\begin{array}{cl}
f(r / s) & \text { if } s \| r \\
0 & \text { otherwise. }
\end{array}\right.
$$


Let now $f, g, h$ be arithmetical functions related by

$$
\sum_{d^{*} \delta=n} f(d) g(\delta)=h(n) .
$$

In the same way that (5) implied (10), (16) implies that

Placing

$$
\sum_{k=1}^{n} f(r ; k) g(k ; s)=h(r ; s), \quad r, s=1,2, \ldots, n .
$$

$$
G(r, s)=\sum_{k=1}^{r} g(k ; s), \quad G(0, s)=0,
$$

and letting $\Sigma$ denote the left member of (17), one obtains by partial summation,

$$
\begin{aligned}
\sum=\sum_{k=1}^{n} f(r ; k)(G(k, s)- & G(k-1, s)) \\
= & \sum_{k=1}^{n}(f(r ; k)-f(r ; k+1)) G(k, s)+f(r ; n+1) G(n, s) .
\end{aligned}
$$

That is,

$$
\sum_{k=1}^{n} f(r ; k)-f(r ; k+1) G(k ; s)=h(r ; s), r, s=1,2, \ldots, n,
$$

or in matric notation,

$$
(f(r ; s)-f(r ; s+1))(G(r, s))=(h(r ; s)), \quad r, s=1, \ldots, n .
$$

\section{Specialisations}

We consider some special cases. Let $\phi^{*}(n)$ denote the number of integers $b(\bmod n)$ such that 1 is the largest unitary divisor of $n$ which divides $b$. In $(2,(2.4))$ it was proved that

$$
\sum_{d \| n} \phi^{*}(d)=n
$$

With $f(n)=\phi^{*}(n), g(n)=\rho(n), h(n)=n,(16)$ reduces to (21), and (20) becomes, by (11),

$$
\left(\phi^{*}(r ; s)-\phi^{*}(r ; s+1)\right)([r, s])=(r \rho(r ; s) / s), \quad r, s=1,2, \ldots, n .
$$

For positive integers $k$, let $L_{k}$ denote the set of integers $n$ whose prime factors all have multiplicity $\geqq k$. Let $L=L_{2}$, so that $L$ is the set of "square-full" integers. Also, let $l_{k}(n)$ denote the characteristic function of $L_{k+1}, l(n)=l_{1}(n)$. In (3, Lemma 3$)$, it was proved that for each positive integer $t$,

$$
\sum_{d \| n} \mu_{t}^{*}(d)=l_{t}(n) \stackrel{\text { def }}{=}\left\{\begin{array}{l}
1\left(n \in L_{t+1}\right) \\
0\left(n \notin L_{t+1}\right),
\end{array}\right.
$$

where $\mu_{t}^{*}(n)$ is the multiplicative function defined for primes $p$ and non-negative integers $e$, by

$$
\mu_{t}^{*}\left(p^{e}\right)=1,-1, \text { or } 0,
$$

according as $e=0,0<e \leqq t$, or $e>t$.

E.M.S.-K 
Placing $f(n)=\mu_{i}^{*}(n), g(n)=\rho(n), h(n)=l_{t}(n)$, (20) yields, by virtue of (16) and (23), the matric relation,

$$
\left.\left(\mu_{i}^{*}(r ; s)-\mu_{i}^{*}(r ; s+1)\right)([r, s])\right)=\left(l_{r}(r ; s)\right), \quad r, s=1,2, \ldots, n .
$$

It will be observed that $\mu_{1}^{*}(n)=\mu(n)$; hence (24) becomes in the case $t=1$,

$$
(\mu(r ; s)-\mu(r ; s+1))([r, s])=(l(r ; s)), \quad r, s=1,2, \ldots, n .
$$

This may be viewed as a second analogue of Jacobsthal's formula. The original one also results from (24), on letting $t \rightarrow \infty$ and noting that

$$
\lim _{t \rightarrow \infty} \mu_{t}^{*}(n)=\mu^{*}(n), \lim _{t \rightarrow \infty} l_{t}(n)=\epsilon(n) .
$$

\section{REFERENCES}

(1) L. Carlitz, Some matrices related to the greatest integer function, Journal of the Elisha Mitchell Scientific Society, 76 (1960), 5-7.

(2) ECKFORD COHEN, Arithmetical functions associated with the unitary divisors of an integer, Mathematische Zeitschrift, 74 (1960), 66-80.

(3) ECKford CoHEn, Arithmetical notes, $X$. A class of totients, to appear in Proc. Amer. Math. Soc.

(4) E. JACOBSThal, Úber die grösste ganze Zahl, II, Norske Videnskabers Selskab Forhandlinger (Trondheim), 30 (1957), 6-13.

Department of Mathematics

UNIVERSITY OF TENNESSEE

KNOXVILLE

TENNESSEE, U.S.A. 